(treatment/prognosis discussions, advance care planning, treatment withdrawal, memory-making, bereavement care) and felt it improved care by providing privacy, removing distractions and facilitating the humanisation of care. However, issues were highlighted that influenced how $\mathrm{MH}$ was understood and used by staff, and have subsequently shaped the routine use of $\mathrm{MH}$. These included issues relating to the practical management of $\mathrm{MH}$, sense of ownership and shared understanding of purpose.

Conclusion The findings reveal important information about how palliative care can be improved in acute settings. They show how initial implementation strategies can influence staff engagement with innovations like $\mathrm{MH}$ and suggest factors that affect uptake and the quality of care. This work is informing the development of $\mathrm{MH}$ and has wider implications for how other acute settings can transform their care environments for the benefit of patients and families.

\section{SERVICE EVALUATION: IDENTIFYING FACTORS CONTRIBUTING TO PROLONGED ADMISSIONS AT MARIE CURIE HOSPICE, NEWCASTLE}

Craig Gouldthorpe, Kym Wakefield, Anne-Marie Bourke. Marie Curie Hospice, Newcastle

10.1136/spcare-2021-PCC.113

Background The average hospice inpatient stay is 15 days [1]. Admissions can extend beyond this for a variety of reasons. We aimed to 1) measure how frequently length of stay (LoS) significantly exceeded this average at Marie Curie Hospice Newcastle, deemed internally as 25 days or longer, and 2) identify factors common to LoS. LoS is relevant to the limited, intensive resource a hospice provides, where a focus is on symptom control and end of life care.

Methods Hospice inpatients admitted for 25 days or more over a nine-month period (March-December 2019) were identified using SystmOne. Reasons for referral, discharge destinations, Phase of Illness (PoI) and Australian Karnofsky performance scores (AKPS) were recorded, and notes were reviewed to identify contributing factors to LoS.

Results Of 181 patients admitted over the 9-month period, 53 patients (29\%) were admitted for 25 days or longer. The median duration of stay was 35 days. Referral reason was multifactorial including symptom control, end of life care, and social breakdown. PoI on admission was unstable (23) and deteriorating (30). AKPS during the admission deteriorated (36), remained static (11) or improved (6).

28 patients died during admission and 25 patients were discharged; the majority $(88 \%)$ to a nursing or care home. The four most common themes contributing to prolonged stays were complex symptoms (28), gradual deterioration (23), acute medical deterioration (21) and awaiting input from other clinical specialties (14).

Conclusions A prolonged inpatient stay was unavoidable for most patients and related to the underlying patient condition, including complex symptom management and variable disease trajectories. This perhaps highlights the complexities and management challenges with this patient cohort. The hospice has implemented changes to reduce LoS including targets for completing fast-track applications, arranging discharge planning meetings, multi-disciplinary team discharge discussions and the documentation of preferred places.

\section{ESTIMATION OF PALLIATIVE CARE NEEDS OF PEOPLE EXPERIENCING HOMELESSNESS USING MORTALITY DATA AND CAUSE-OF-DEATH}

1,2 Aoibheann Conneely, ${ }^{3}$ Jo-Hanna Ivers, ${ }^{3} J$ Joe Barry, ${ }^{4}$ Elaine Dunne, 1,2,5 Norma O'Lean

${ }^{1,6}$ Cliona Ni Cheallaigh. 'School of Medicine, Trinity College Dublin, Ireland; ${ }^{2}$ Academic Department of Palliative Medicine, Our Lady's Hospice and Care Services, Harold's Cross, Dublin, Ireland; ${ }^{3}$ Department of Public Health and Primary Care, Institute of Population Health, School of Medicine, Trinity College Dublin, Ireland; ${ }^{4}$ Hospital Inpatient Enquiry Department, St James's hospital, Dublin, Ireland; ${ }^{5}$ Department of Palliative Medicine, St James's Hospital, Dublin, Ireland; ${ }^{6}$ Department of General Medicine and Infectious Diseases, St James's Hospital, Dublin, Ireland

\subsection{6/spcare-2021-PCC.114}

Background People experiencing homelessness have higher standardised mortality rates and higher risk of death than the general population. In recent years, inclusion health services have developed internationally with the aim of addressing extreme health and social inequities. Much of the research exploring delivery of palliative care to people experiencing homelessness is qualitative. We are unaware of a study that has attempted to quantify the palliative care needs of people experiencing homelessness.

Aim To apply cause-of-death based methodology of estimating palliative care needs to deaths of people experiencing homelessness. To evaluate the appropriateness of this methodology to quantify the palliative care needs of this population.

Design Secondary data analysis of a previously reported observational study. Mortality data (2005-2015) of people experiencing homelessness was reviewed for illnesses (malignant and non-malignant) potentially requiring palliative care input, using specific International Classification of Diseases-10 codes. This method, developed by Murtagh et al, has been used widely internationally to calculate both the current and future palliative care needs of populations. Setting and participants: $\mathrm{N}=361$ deaths of people experiencing homelessness in Dublin between 2005-2015.

Results A minimum of $25 \%$ of all deaths of people experiencing homelessness would have benefitted from palliative care. This percentage increases according to age-group with at least $64 \%$ of people experiencing homelessness over the age of 60 years dying of illnesses which would benefit from palliative care input.

Conclusions Estimating palliative care needs based on single underlying-cause of death has limitations when applying causeof-death based methodology to a population of people experiencing homelessness. Incorporating multimorbidity would provide a more accurate estimate of need. In developing a palliative care response to inclusion health, older people in homelessness should be considered a priority group.

\section{PROSPECTIVE AUDIT OF PATIENTS ADMITTED TO A NEWLY ESTABLISHED SUPPORTIVE CARE UNIT IN WIRRAL UNIVERSITY TEACHING HOSPITAL (WUTH)}

Bethan Roberts, Rachel Parry, Richard Latten, Clare Jeffries, Catherine Hayle. Wirral University Teaching Hospital (WUTH)

\subsection{6/spcare-2021-PCC.115}

Background Following redesign of palliative care services, four specialist palliative care beds were opened in August 2019 on a ward shared with Haematology. An SOP was developed to guide admissions to the Supportive Care Unit (SCU), 
prioritised from the advisory caseload as patients with predominantly palliative care needs, requiring regular consultant input.

Aims Prospective audit of SCU admissions during the initial nine-month period from opening in August 2019.

Results Over the initial nine-month period, a total of 85 patients were admitted to SCU with a mean length of stay of 10 days. Of these, $86 \%(n=72)$ had a primary cancer diagnosis, compared with $64 \%$ of patients under our advisory caseload. Age range was 37-98 years, with a mean age of 67 years. Mean waiting time once listed was 2 days (median 1 day, range 0-32 days). Mean time from referral to the advisory HSPCT to referral to SCU was 5 days (median 1 day, range 0-88 days). Length of stay reduced from a mean of 15 days in month one to 7 days in month six. Patients were most commonly admitted from medical wards (58\%), followed by assessment areas (32\%) and surgical wards (5\%). Four patients were admitted electively; two for blood transfusion and two for paracentesis.

Conclusion Without any additional resource, we have successfully opened a small number of specialist palliative care beds. In September 2020, this increased to eight beds. Informal feedback is positive, and our next objective is to capture formal patient and family feedback. We will also develop processes to facilitate elective admissions for patients already known to palliative care services. It is interesting to note that the proportion of patients with a cancer diagnosis is higher on SCU compared with the advisory HSPCT. Prospective data collection is ongoing to see if this trend continues.

\section{IMPROVING USE OF CO-ORDINATE MY CARE ELECTRONIC PATIENT RECORD AT ST CHRISTOPHER'S HOSPICE - COMPLETING THE AUDIT CYCLE}

Christopher Doyle, Joy Ross. St Christopher's Hospice

\subsection{6/spcare-2021-PCC.116}

Introduction Co-ordinate my care $(\mathrm{CMC})$ is the pan-london electronic patient record system for palliative care patients. It facilitates sharing urgent care records across settings, linking services which patients may access(GP, ambulance, district nurses, palliative care). By creating records and working with local services to update records, we aim to improve 'joined up working' for our patients.

Aims/objectives To audit use of CMC for patients under the hospice including numbers of patient consented/records created, congruence of information recorded with hospice electronic records and access of records by emergency services.

Methods We conducted snapshot audits of records in december 2018, subsequent QI initiatives including staff training and integration of CMC reviews into MDM meetings, with re-audit December 2019. Each audit examined 200 patient records, 100 indicated as 'CMC record created' on our hospice system and 100 indicated to have no record. Patients were randomly selected; 40 from each service:Bromley community, bromley care co-ordination, croydon community, Lambeth/southwark/lewisham community and sydenham outpatients. Information was crosschecked against the live CMC record. Standards included: $90 \%$ of patients should have a CMC record, $95 \%$ should have documentation of consideration of CMC, 95\% of CMC records should have resuscitation and ceilings of care decisions recorded with congruence between systems We also recorded whether CMC records were accessed by $\mathrm{OOH}$ services.

Results Comparing data from our initial audit to the re-audit; $61 \%$ vs $73 \%$ of patients under our care were consented to have records created. Of these $>95 \%$ had a live CMC record. For those not consented by us $220-40 \%$ had a live record across each of the 5 services. Between audits, numbers of patients with CMC records increased from 48-60\% to 58$68 \%$. Discussion of CMC with patients increased from 48 $53 \%$ to $70-93 \%$. Recording of DNAR status averaged $>95 \%$. Congruence of DNAR decisions was $82 \%$ with ceilings of care $61 \%$ congruent. Inclusion of hospice contact details varied across teams (30 to 74\%). Records were accessed by out of hours services for 13 of these patients.

Conclusion Use of $\mathrm{CMC}$ is increasing with access to records by $\mathrm{OOH}$ services. Further QI initiatives to drive numbers and quality of records will be discussed with impact of $\mathrm{OOH}$ records accessed on patient outcome.

\section{9 'TO FEED OR NOT TO FEED': REVIEW OF PRACTICE IN AN ACUTE HOSPITAL TRUST AROUND INITIATION AND WITHDRAWAL OF PARENTERAL NUTRITION (PN) IN PALLIATIVE PATIENTS}

David J Matthews, Farida Malik. East Sussex Healthcare NHS Trust

\subsection{6/spcare-2021-PCC.117}

Background/Introduction National and European guidance around use of PN in palliative patients emphasises the need to consider risks, benefits, and impact on QOL with it unlikely to be appropriate in the last weeks of life. Starting PN can delay discharge from hospital to other settings and affect PPC/ PPD.

Aims/Objectives To assess whether local processes around initiating and withdrawing PN for hospital inpatients with palliative care needs are in line with current guidance.

Methods Retrospective case review: All Patients prescribed PN feed over a 6-month period identified by pharmacy and known to the hospital supportive and palliative care team (SPCT) were recruited and their notes reviewed regarding indication for $\mathrm{PN}$, evidence of discussions around initiation and withdrawal, date PN started and stopped, interventions planned/performed, details of SPCT involvement, and patient outcomes. Data analysed using descriptive statistics.

Results 183 patients received PN over 6-month period. 33 were known to SPCT. SPCT involvement began after PN initiation for $77 \%$ of patients. Wide range of indications for initiation, most common being oesophageal obstruction (16\%). Most commonly withdrawn because patient approaching terminal phase (52\%.) Relevant documented discussions with patients/families recorded in $29 \%$ prior to initiating PN (none involved SPCT) and 26\% prior to withdrawing PN (half involving SPCT). Nearly half (48\%) of patients died within 2 weeks of starting PN (65\% within 4 weeks). Importantly $26 \%$ died within $24 \mathrm{hrs}$ of stopping PN. Over $2 / 3$ died in hospital. $65 \%$ had no intervention planned to stop the need for ongoing $\mathrm{PN}$ at the time of initiation. Findings showed results were not in line with current national guidance

Conclusion/Discussion Decision for SPCT to be involved in all MDT discussions prior to $\mathrm{PN}$ initiation in palliative patients 